TRANSACTIONS OF THE

AMERICAN MATHEMATICAL SOCIETY

Volume 350, Number 4, April 1998, Pages 1407-1428

S 0002-9947(98)02140-0

\title{
THE BEHAVIOR OF THE HEAT OPERATOR ON WEIGHTED SOBOLEV SPACES
}

\author{
G. N. HILE AND C. P. MAWATA
}

\begin{abstract}
Denoting by $\mathcal{H}$ the heat operator in $R^{n+1}$, we investigate its properties as a bounded operator from one weighted Sobolev space to another. Our main result gives conditions on the weights under which $\mathcal{H}$ is an injection, a surjection, or an isomorphism. We also describe the range and kernel of $\mathcal{H}$ in all the cases. Our results are analogous to those obtained by R. C. McOwen for the Laplace operator in $R^{n}$.
\end{abstract}

\section{INTRODUCTION}

Let $\mathcal{H}$ denote the heat operator in $\mathbb{R}^{n+1}=\mathbb{R}^{n} \times \mathbb{R}, n \geq 1$,

$$
\mathcal{H}:=\partial_{t}-\Delta_{x} \text {. }
$$

We investigate properties of $\mathcal{H}$ as a bounded operator

$$
\mathcal{H}: P_{\delta}^{2,1, p} \rightarrow P_{\delta+2}^{p},
$$

where the Banach spaces $P_{\delta}^{2,1, p}$ and $P_{\delta}^{p}$, and the spaces of polynomials $H_{m}$, are defined as follows (all functions real or extended real valued):

Definition. Let $\rho$ be a weight function $\rho(x, t):=\sqrt{1+|x|^{2}+|t|}$; we define

(i) $P_{\delta}^{p}$ is the space of functions $u$ on $\mathbb{R}^{n+1}$ with finite norm

$$
\|u\|_{p ; \rho, \delta}:=\left\|\rho^{\delta} u\right\|_{p},
$$

(ii) $P_{\delta}^{2,1, p}$ is the space of functions $u$ on $\mathbb{R}^{n+1}$ with finite weighted Sobolev norm

$$
\|u\|_{2,1, p ; \rho, \delta}:=\sum_{|\alpha| \leq 2}\left\|\rho^{\delta+|\alpha|} D_{x}^{\alpha} u\right\|_{p}+\left\|\rho^{\delta+2} D_{t} u\right\|_{p},
$$

(iii) $H_{m}$ is the space of all polynomial solutions of the heat equation in $\mathbb{R}^{n+1}$, of degree at most $m$ in the space variable $x$.

Our results can be summarized in the following theorem (where $\mathbb{N}$ denotes the collection of nonnegative integers):

Theorem 1.1. Suppose $1<p<\infty$ and $\delta \in \mathbb{R}, m \in \mathbb{N}$, and consider the mapping $(\dagger)$.

(a) If $-(n+2) / p<\delta<n-(n+2) / p$, then ( $\dagger)$ is an isomorphism.

Received by the editors April 12, 1996

1991 Mathematics Subject Classification. Primary 35B45; Secondary 35A05, 35J60.

(C)1998 American Mathematical Society 
(b) If $m+n-(n+2) / p<\delta<1+m+n-(n+2) / p$, then ( $\dagger$ ) is one-to-one, and the range of $\mathcal{H}$ is the closed subspace

$$
\left\{f \in P_{\delta+2}^{p}: \int_{\mathbb{R}^{n+1}} f(x, t) h(x,-t) d x d t=0, \forall h \in H_{m}\right\} .
$$

(c) If $-1-m-(n+2) / p<\delta<-m-(n+2) / p$, then ( $\dagger)$ is onto, and the kernel of $\mathcal{H}$ is $H_{m}$.

(d) If $\delta=-m-(n+2) / p$, then the kernel of $\mathcal{H}$ is $H_{m-1}$, and the range of $\mathcal{H}$ is not closed.

(e) If $\delta=m+n-(n+2) / p$, then $(\dagger)$ is one-to-one, and the range of $\mathcal{H}$ is not closed.

It follows in particular that $(\dagger)$ describes a Fredholm mapping in cases (a), (b), and (c), but not in (d) and (e); moreover, the Fredholm index of $\mathcal{H}$ is 0 in case (a), $-\operatorname{dim} H_{m}$ in (b), and $\operatorname{dim} H_{m}$ in (c).

These results are analogous to those of McOwen [11] for the Laplace operator $\Delta$ in $\mathbb{R}^{n}$. McOwen investigated $\Delta$ as an operator

$$
\Delta: M_{2, \delta}^{p} \rightarrow L_{\delta+2}^{p},
$$

where the spaces $M_{2, \delta}^{p}$ and $L_{\delta+2}^{p}$ have respective norms

$$
\sum_{|\alpha| \leq 2}\left\|\omega^{\delta+|\alpha|} D_{x}^{\alpha} u\right\|_{p}, \quad\left\|\omega^{\delta+2} u\right\|_{p}
$$

with weight function $\omega(x)=\sqrt{1+|x|^{2}}$. These spaces were first introduced by M. Cantor [2], who gave some sufficient conditions for the mapping $(*)$ to be an isomorphism. McOwen gave a much more complete analysis of $\left(^{*}\right)$, proving a theorem directly analogous to our Theorem 1.1. We borrow liberally from McOwen's techniques in this paper.

Results like Theorem 1.1 were proved for elliptic operators, and elliptic systems of operators, by Lockhart and McOwen [8], [9], [12]. Such operators are allowed variable coefficients, but the coefficients of the highest order terms must approach constants at infinity at a suitable rate. These authors were influenced by earlier papers of Nirenberg and Walker [13], [14], [15], who studied the null spaces of elliptic operators on Sobolev spaces $W^{m, p}\left(\mathbb{R}^{n}\right)$.

For related results for parabolic equations of second order, both linear and nonlinear, see [6], [10]. In these papers the authors showed that, under certain hypotheses, solutions of polynomial growth of a parabolic equation behave at infinity like polynomial solutions of the heat equation. Similar statements were established for linear and nonlinear elliptic equations of second order in [1] and [5].

\section{Preliminaries}

We let $x=\left(x_{1}, x_{2}, \cdots, x_{n}\right)$ denote a point in $\mathbb{R}^{n}, n \geq 1$, we let $t$ denote a point in $\mathbb{R}$, and $(x, t)=\left(x_{1}, x_{2}, \cdots, x_{n}, t\right)$ a point in $\mathbb{R}^{n+1}=\mathbb{R}^{n} \times \mathbb{R}$. We let $\rho$ and $\sigma$ denote functions on $\mathbb{R}^{n+1}$ prescribed by

$$
\rho(x, t)=\sqrt{1+|x|^{2}+|t|}, \sigma(x, t)=\sqrt{|x|^{2}+|t|} .
$$

As is well known, [3, chapter 3], the quantity $d((x, t),(y, s)):=\sigma(x-y, t-s)$ is a metric on $\mathbb{R}^{n+1}$; in particular, we have the triangle inequalities

$$
\sigma(x, t)-\sigma(y, s) \leq \sigma(x-y, t-s) \leq \sigma(x, t)+\sigma(y, s) .
$$


Note also that $\sigma<\rho$ and $1 \leq \rho \leq 1+\sigma$.

All functions in this paper are real or extended real valued, defined on all of $\mathbb{R}^{n+1}=\mathbb{R}^{n} \times \mathbb{R}$. Let $u(x, t)$ be such a function. Given a multi-index $\alpha$ in $\mathbb{R}^{n}$ and a nonnegative integer $k$, by $D_{x}^{\alpha} u$ and $D_{t}^{k} u$ we mean the Sobolev derivatives

$$
D_{x}^{\alpha} u=\partial_{1}^{\alpha_{1}} \partial_{2}^{\alpha_{2}} \cdots \partial_{n}^{\alpha_{n}} u, \quad D_{t}^{k} u=\partial_{n+1}^{k} u=\partial^{k} u / \partial t^{k} .
$$

For $1 \leq p \leq \infty,\|u\|_{p}$ denotes the usual $L^{p}$ norm of $u$ over $\mathbb{R}^{n+1}$.

Definition. For nonnegative integers $m$ and $k$, and for $\delta \in \mathbb{R}, 1 \leq p \leq \infty$,

a) the space $W_{\text {loc }}^{m, k, p}\left(\mathbb{R}^{n+1}\right)$ is the collection of functions $u$ on $\mathbb{R}^{n+1}$ for which the space derivatives $D_{x}^{\alpha} u, 0 \leq|\alpha| \leq m$, and time derivatives $D_{t}^{j} u, 0 \leq j \leq k$, are in $L_{\mathrm{loc}}^{p}\left(\mathbb{R}^{n+1}\right)$;

b) the space $P_{\delta}^{m, k, p}$ is the collection of functions $u$ in $W_{\mathrm{loc}}^{m, k, p}\left(\mathbb{R}^{n+1}\right)$ with finite weighted norm

$$
\|u\|_{m, k, p ; \rho, \delta}:=\sum_{|\alpha| \leq m}\left\|\rho^{\delta+|\alpha|} D_{x}^{\alpha} u\right\|_{p}+\sum_{j=1}^{k}\left\|\rho^{\delta+2 j} D_{t}^{j} u\right\|_{p} .
$$

(When $k=0$, the second summation is vacuous.)

Note that $P_{\delta}^{p}$ (as defined in the introduction) is $P_{\delta}^{0,0, p}$; also note that

$$
\delta_{1} \leq \delta_{2} \Rightarrow P_{\delta_{2}}^{m, k, p} \subset P_{\delta_{1}}^{m, k, p} .
$$

The spaces $P_{\delta}^{m, k, p}$ are analogous to the weighted Sobolev spaces $M_{s, \delta}^{p}$ introduced by M. Cantor [2] and utilized by Lockhart and McOwen [8], [9], [11], [12]. These authors, in working with elliptic operators instead of the heat operator, used the weight function $\left(1+|x|^{2}\right)^{1 / 2}$ instead of our $\rho(x, t)$.

We require the following technical lemma; its proof detracts from our main development and is delayed until the last section.

Lemma 2.1. For given integers $m \geq 0, k \geq 0$, and for $1 \leq p<\infty, \delta \in \mathbb{R}$, the space $C_{0}^{\infty}\left(\mathbb{R}^{n+1}\right)$ is dense in $P_{\delta}^{m, k, p}$.

We let $\langle u, v\rangle$ denote the usual inner product of functions $u$ and $v$ on $\mathbb{R}^{n+1}$;

$$
\langle u, v\rangle:=\int_{\mathbb{R}^{n+1}} u v d x d t .
$$

When $1 \leq p \leq \infty$ the number $q$ will be determined by $1 / p+1 / q=1$. For $u \in P_{\delta}^{p}$ and $v \in P_{-\delta}^{q}$, Hölder's inequality confirms that

$$
|\langle u, v\rangle| \leq\|u\|_{p ; \rho, \delta}\|v\|_{q ; \rho,-\delta} .
$$

Indeed, it is easily argued that, when $1<p<\infty$, the spaces $P_{\delta}^{p}$ and $P_{-\delta}^{q}$ are duals of one another.

We consider the operator $\mathcal{H}$ on $\mathbb{R}^{n+1}$, as defined by

$$
\mathcal{H} u:=u_{t}-\Delta_{x} u=\frac{\partial u}{\partial t}-\sum_{i=1}^{n} \frac{\partial^{2} u}{\partial x_{i}^{2}} .
$$

We interpret $\mathcal{H}$ in the distributional sense; that is, for functions $u$ and $f$ in $L_{\text {loc }}^{1}\left(\mathbb{R}^{n+1}\right)$ we say that $\mathcal{H} u=f$ in $\mathbb{R}^{n+1}$ provided that, for all functions $\phi$ in 
$C_{0}^{\infty}\left(\mathbb{R}^{n+1}\right)$,

$$
\int_{\mathbb{R}^{n+1}} u\left(-\phi_{t}-\Delta \phi\right) d x d t=\int_{\mathbb{R}^{n+1}} f \phi d x d t .
$$

We define $u^{*}$ (and analogously $f^{*}$ and $\phi^{*}$ ) on $\mathbb{R}^{n+1}$ according to

$$
u^{*}(x, t):=u(x,-t)
$$

then, making the change of parameter $t \rightarrow-s$ in (2.5), and replacing $\phi$ by $\phi^{*}$, we discover the equivalent formulation

$$
\mathcal{H} u=f \text { in } \mathbb{R}^{n+1} \Leftrightarrow\left\langle u^{*}, \mathcal{H} \phi\right\rangle=\left\langle f, \phi^{*}\right\rangle, \forall \phi \in C_{0}^{\infty}\left(\mathbb{R}^{n+1}\right) .
$$

We denote by $K_{n}$ the fundamental solution for the heat equation in $\mathbb{R}^{n+1}$,

$$
K_{n}(x, t):= \begin{cases}(4 \pi t)^{-n / 2} e^{-|x|^{2} / 4 t}, & \text { if } t>0 \\ 0, & \text { if } t \leq 0 .\end{cases}
$$

When there is no risk of ambiguity we shall write $K_{n}$ as $K$. Note that

$$
K_{n}(x, t)=\prod_{i=1}^{n} K_{1}\left(x_{i}, t\right) .
$$

For appropriate functions $f$ on $\mathbb{R}^{n+1}$, the convolution $\mathcal{K} f$ is defined by

$$
\mathcal{K} f(x, t):=\int_{\mathbb{R}^{n+1}} K(x-y, t-s) f(y, s) d y d s .
$$

As is well known, if $\phi \in C_{0}^{\infty}\left(\mathbb{R}^{n+1}\right)$, then $\mathcal{K} \phi \in C^{\infty}\left(\mathbb{R}^{n+1}\right)$ and $\mathcal{H}(\mathcal{K} \phi)=\phi$.

We shall also make use of an integral operator $\mathcal{S}$, given by

$$
\mathcal{S} f(x, t):=\int_{\mathbb{R}^{n+1}} \sigma(x-y, t-s)^{-n} f(y, s) d y d s .
$$

The proof of the following lemma, quite computational in nature, is likewise postponed until the last section:

Lemma 2.2. Assume

$$
1 \leq p \leq \infty, \quad-\frac{n+2}{p}<\delta<n-\frac{n+2}{p} .
$$

If $f \in P_{\delta+2}^{p}$, then $\mathcal{K} f, \mathcal{S} f \in P_{\delta}^{p}$ and

$$
\|\mathcal{K} f\|_{p ; \rho, \delta},\|\mathcal{S} f\|_{p ; \rho, \delta} \leq C(n, p, \delta)\|f\|_{p ; \rho, \delta+2} .
$$

We require a special case of a theorem for more general parabolic operators, from the authors' earlier paper [6]:

Lemma 2.3. If $1<p<\infty$ and $\delta \in \mathbb{R}$, then, for all $u$ in $W_{\mathrm{loc}}^{2,1, p}\left(\mathbb{R}^{n+1}\right)$,

$$
\|u\|_{2,1, p ; \rho, \delta} \leq C(n, p, \delta)\left[\|u\|_{p ; \rho, \delta}+\|\mathcal{H} u\|_{p ; \rho, \delta+2}\right] .
$$

Proof. The result is a special case of Lemma 1B from [6].

Theorem 2.4. Suppose inequalities (2.8) hold. If $f \in P_{\delta+2}^{p}$, then $\mathcal{K} f \in P_{\delta}^{2,1, p}$, with $\mathcal{H}(\mathcal{K} f)=f$ and

$$
\|\mathcal{K} f\|_{2,1, p ; \rho, \delta} \leq C(n, p, \delta)\|f\|_{p ; \rho, \delta+2} .
$$


Proof. Let $\left\{\phi_{l}\right\}$ be a sequence in $C_{0}^{\infty}\left(\mathbb{R}^{n+1}\right)$ converging to $f$ in $P_{\delta+2}^{p}$. Then each $\mathcal{K} \phi_{l}$ is in $C_{0}^{\infty}\left(\mathbb{R}^{n+1}\right)$, with

$$
\mathcal{H}\left(\mathcal{K} \phi_{l}\right)=\phi_{l}
$$

Lemma 2.2 gives

$$
\left\|\mathcal{K} \phi_{l}\right\|_{p ; \rho, \delta} \leq C(n, p, \delta)\left\|\phi_{l}\right\|_{p ; \rho, \delta+2}
$$

and then Lemma 2.3, applied to $u=\mathcal{K} \phi_{l}$, leads to

$$
\left\|\mathcal{K} \phi_{l}\right\|_{2,1, p ; \rho, \delta} \leq C(n, p, \delta)\left\|\phi_{l}\right\|_{p ; \rho, \delta+2} .
$$

Moreover, (2.14) holds for each difference $\phi_{l}-\phi_{m}$, and (2.13) for each difference $\phi_{l}-f$. These observations lead to the conclusion that the sequence $\left\{\mathcal{K} \phi_{l}\right\}$ is Cauchy in $P_{\delta}^{2,1, p}$, and thereby that $\mathcal{K} f \in P_{\delta}^{2,1, p}$ with $\mathcal{K} \phi_{l}$ converging to $\mathcal{K} f$ in the norm of this space. Letting $l \rightarrow \infty$ in (2.14) and (2.12) yields (2.11) as well as $\mathcal{H}(\mathcal{K} f)=f$.

Corollary 2.5. Suppose $f \in P_{\delta+2}^{p}$ and $u \in P_{\delta}^{p}$, where $1<p<\infty$ and $\delta \in \mathbb{R}$. If $u$ is a distributional solution in $\mathbb{R}^{n+1}$ of $\mathcal{H} u=f$, then $u \in P_{\delta}^{2,1, p}$.

Proof. Let $\mathcal{B}$ be any open ball in $\mathbb{R}^{n+1}$. Since $f \chi_{\mathcal{B}} \in P_{\epsilon}^{p}$ for all $\epsilon>0$, Theorem 2.4 asserts that $w:=\mathcal{K}\left(f \chi_{\mathcal{B}}\right) \in W_{\text {loc }}^{2,1, p}\left(\mathbb{R}^{n+1}\right)$ with $\mathcal{H} w=f \chi_{\mathcal{B}}$. Thus $u-w$, a distributional solution in $\mathcal{B}$ of the heat equation, is in $C^{\infty}(\mathcal{B})$. Since $\mathcal{B}$ is arbitrary, $u \in W_{\mathrm{loc}}^{2,1, p}\left(\mathbb{R}^{n+1}\right)$. By (2.10) of Lemma $2.3, u \in P_{\delta}^{2,1, p}$.

\section{The Isomorphism Range}

We verify statement (a) of Theorem 1.1 .

Lemma 3.1. Suppose $1<p<\infty$ and $\delta \in \mathbb{R}$.

a) If $u \in P_{\delta}^{2,1, p}$ and $v \in P_{-\delta-2}^{2,1, q}$, then

$$
\left\langle\mathcal{H} u, v^{*}\right\rangle=\left\langle u^{*}, \mathcal{H} v\right\rangle \text {. }
$$

b) If $u \in P_{\delta}^{2,1, p}$ and $w \in P_{-\delta}^{q}$, and if $-(n+2) / p<\delta<n-(n+2) / p$, then $\mathcal{K} w \in P_{-\delta-2}^{2,1, q}$ and

$$
\left\langle\mathcal{H} u,(\mathcal{K} w)^{*}\right\rangle=\left\langle u^{*}, w\right\rangle .
$$

Proof. To prove (a), let $\left\{\phi_{l}\right\}$ be a sequence in $C_{0}^{\infty}\left(\mathbb{R}^{n+1}\right)$ converging to $v$ in the space $P_{-\delta-2}^{2,1, q}$. By $(2.6)$, for each $l$ we have

$$
\left\langle\mathcal{H} u, \phi_{l}^{*}\right\rangle=\left\langle u^{*}, \mathcal{H} \phi_{l}\right\rangle .
$$

Since $\mathcal{H} u \in P_{\delta+2}^{p}$ and $\phi_{l}^{*} \rightarrow v^{*}$ in $P_{-\delta-2}^{q}$, while $u^{*} \in P_{\delta}^{p}$ and $\mathcal{H} \phi_{l} \rightarrow \mathcal{H} v$ in $P_{-\delta}^{q}$, we may take limits in (3.3) to obtain (3.1).

To prove (b), we first observe that

$$
-\frac{n+2}{p}<\delta<n-\frac{n+2}{p} \Leftrightarrow-\frac{n+2}{q}<-\delta-2<n-\frac{n+2}{q} .
$$

Thus we may apply Theorem 2.4 to $w$, with $p$ replaced by $q$ and $\delta$ by $-\delta-2$, and conclude that $\mathcal{K} w \in P_{-\delta-2}^{2,1, q}$ with $\mathcal{H}(\mathcal{K} w)=w$. Substitution of $\mathcal{K} w$ for $v$ in (3.1) then gives (3.2). 
Theorem 3.2. Assume $1<p<\infty$ and $-(n+2) / p<\delta$. Then

$$
\mathcal{H}: P_{\delta}^{2,1, p} \rightarrow P_{\delta+2}^{p}
$$

is one-to-one. If moreover $\delta<n-(n+2) / p$, then $\mathcal{H}$ is onto, and hence an isomorphism.

Proof. Suppose $u \in P_{\delta}^{2,1, p}$ with $\mathcal{H} u=0$ and $-(n+2) / p<\delta$. We may decrease $\delta$, if necessary, so that also $\delta<n-(n+2) / p$; then Lemma 3.1(b) shows that $\left\langle u^{*}, w\right\rangle=0$ for all $w$ in $P_{-\delta}^{q}$, and hence that $u^{*}=0, u=0$. Thus $\mathcal{H}$ is one-to-one. Next if $f \in P_{\delta+2}^{p}$, and (2.8) holds, Theorem 2.4 asserts that $\mathcal{K} f \in P_{\delta}^{2,1, p}$ and $\mathcal{H}(\mathcal{K} f)=f$; thus $\mathcal{H}$ is onto.

\section{Heat POLynomials}

The basic theory of the so-called heat polynomials was developed by Widder [16], [17]. In one space dimension the heat polynomials $v_{l}, l \in \mathbb{N}$, are certain polynomial solutions of the heat equation, as prescribed by

$$
v_{l}(x, t):=l ! \sum_{k=0}^{\lfloor l / 2\rfloor} \frac{x^{l-2 k} t^{k}}{k !(l-2 k) !} .
$$

(Here $\lfloor z\rfloor$ denotes the greatest integer not exceeding $z$.) The heat polynomials in $n$ space dimensions are subscripted with multi-indices $\beta=\left(\beta_{1}, \beta_{2}, \cdots, \beta_{n}\right)$, and can be defined in terms of heat polynomials in one space dimension as the product

$$
v_{\beta}(x, t):=\prod_{i=1}^{n} v_{\beta_{i}}\left(x_{i}, t\right) .
$$

We shall denote by $\mathbb{N}^{n}$ the space of all such multi-indices. For multi-indices $\beta$ and $\gamma$ we say that $\gamma \leq \beta$ iff $\gamma_{i} \leq \beta_{i}$ for each $i$; we also write $x^{\beta}=x_{1}^{\beta_{1}} x_{2}^{\beta_{2}} \cdots x_{n}^{\beta_{n}}$, $|\beta|=\beta_{1}+\beta_{2}+\cdots+\beta_{n}, \beta !=\beta_{1} ! \beta_{2} ! \cdots \beta_{n}$ ! . Then, using (4.1) and some algebra, we can write (4.2) alternatively as

$$
v_{\beta}(x, t)=\beta ! \sum_{2 \gamma \leq \beta} \frac{x^{\beta-2 \gamma} t^{|\gamma|}}{\gamma !(\beta-2 \gamma) !} .
$$

Each $v_{\beta}$ solves the $n$-dimensional heat equation, and, as easily checked, obeys the modified homogeneity condition

$$
v_{\beta}\left(\lambda x, \lambda^{2} t\right)=\lambda^{|\beta|} v_{\beta}(x, t),
$$

valid for $(x, t)$ in $\mathbb{R}^{n+1}$ and any constant $\lambda>0$. From (4.3) we have $v_{\beta}(x, 0)=x^{\beta}$. This equation determines a one-to-one correspondence between heat polynomials $v_{\beta}$ in $\mathbb{R}^{n+1}$ and monomials $x^{\beta}$ in $\mathbb{R}^{n}$.

As described in the introduction, for a given nonnegative integer $m$ the space $H_{m}$ consists of all real polynomial solutions of the heat equation in $\mathbb{R}^{n+1}$ of degree at most $m$ in $x$. (It is shown in [10] that if a polynomial solution is of degree at most $m$ in $x$, then it is of degree at most $\lfloor m / 2\rfloor$ in $t$.) Given $P$ in $H_{m}, P(x, 0)$ takes the form

$$
P(x, 0)=\sum_{|\beta| \leq m} a_{\beta} x^{\beta}
$$


for some real constants $\left\{a_{\beta}\right\}$. But then the polynomial

$$
Q(x, t)=\sum_{|\beta| \leq m} a_{\beta} v_{\beta}(x, t)
$$

solves the heat equation and agrees with $P$ on $t=0$; thus $Q=P$. This argument demonstrates that the heat polynomials $\left\{v_{\beta}:|\beta| \leq m\right\}$ form a vector space basis for $H_{m}$. Thus the dimension of $H_{m}$ is the same as the number of multi-indices in $\mathbb{R}^{n}$ of magnitude no larger than $m$; that is,

$$
\text { dimension } H_{m}=\left(\begin{array}{c}
m+n \\
n
\end{array}\right)=\frac{(m+n) !}{m ! n !} .
$$

Lemma 4.1. Let $\beta$ be any multi-index in $\mathbb{R}^{n}$; then for all $(x, t)$ in $\mathbb{R}^{n+1}$,

$$
\begin{gathered}
\left|v_{\beta}(x, t)\right| \leq C(\beta) \sigma(x, t)^{|\beta|}, \\
\left|\frac{\partial}{\partial x_{i}} v_{\beta}(x, t)\right| \leq \beta_{i} C(\beta) \sigma(x, t)^{|\beta|-1}, \quad i=1,2, \cdots, n .
\end{gathered}
$$

Moreover, if $1<p<\infty$ and $\delta \in \mathbb{R}$, then

$$
v_{\beta} \in P_{\delta}^{2,1, p} \Leftrightarrow v_{\beta} \in P_{\delta}^{p} \Leftrightarrow \delta<-|\beta|-\frac{n+2}{p} .
$$

Proof. We obtain (4.6) and (4.7) from (4.3) and the inequalities $\left|x_{i}\right| \leq \sigma(x, t)$, $|t| \leq \sigma(x, t)^{2}$.

Since $\mathcal{H} v_{\beta}=0$, Lemma 2.3 implies that $v_{\beta} \in P_{\delta}^{2,1, p}$ iff $v_{\beta} \in P_{\delta}^{p}$. First assume $\delta<-|\beta|-(n+2) / p$; we show that $v_{\beta} \in P_{\delta}^{p}$ by using (4.6) with the estimates

$$
\begin{aligned}
\left(\left\|v_{\beta}\right\|_{p ; \rho, \delta}\right)^{p} & =\int_{\mathbb{R}^{n+1}} \rho(x, t)^{\delta p}\left|v_{\beta}(x, t)\right|^{p} d x d t \\
& \leq C(p, \beta) \int_{\mathbb{R}^{n+1}} \rho(x, t)^{\delta p} \sigma(x, t)^{|\beta| p} d x d t \\
& \leq C(p, \beta) \int_{\mathbb{R}^{n}} 2 \int_{0}^{\infty}\left(1+|x|^{2}+t\right)^{(\delta+|\beta|) p / 2} d t d x \\
& =C(p, \beta, \delta) \int_{\mathbb{R}^{n}}\left(1+|x|^{2}\right)^{1+(\delta+|\beta|) p / 2} d x<\infty .
\end{aligned}
$$

Next we assume that $v_{\beta} \in P_{\delta}^{p}$ and show that the right inequality of (4.8) must hold. By the notation $x \geq 0$ we mean that $x_{i} \geq 0$ for $1 \leq i \leq n$. Since all coefficients in (4.3) are nonnegative, when $x \geq 0$ and $t \geq 0$ the summation (4.3) is bounded below by the single term $x^{\beta}$, occurring when $\gamma=0$; thus the first equation in (4.9) gives

$$
\left(\left\|v_{\beta}\right\|_{p ; \rho, \delta}\right)^{p} \geq \int_{1}^{\infty} \int_{x \geq 0} \rho(x, t)^{\delta p}\left(x^{\beta}\right)^{p} d x d t
$$

If $\delta p \geq 0$, then we obtain

$$
\left(\left\|v_{\beta}\right\|_{p ; \rho, \delta}\right)^{p} \geq \int_{1}^{\infty} \int_{x \geq 0}\left(x^{\beta}\right)^{p} d x d t=\infty ;
$$

thus we must have $\delta p<0$. If $t \geq 1$, then $\rho(x, t) \leq\left(|x|^{2}+2 t\right)^{1 / 2}$, giving

$$
\left(\left\|v_{\beta}\right\|_{p ; \rho, \delta}\right)^{p} \geq \int_{1}^{\infty} \int_{x \geq 0}\left(|x|^{2}+2 t\right)^{\delta p / 2}\left(x^{\beta}\right)^{p} d x d t
$$


In the inner integral we substitute $x=\sqrt{t} y, d x=t^{n / 2} d y$, obtaining

$$
\left(\left\|v_{\beta}\right\|_{p ; \rho, \delta}\right)^{p} \geq \int_{1}^{\infty} t^{(\delta p+|\beta| p+n) / 2} \int_{y \geq 0}\left(|y|^{2}+2\right)^{\delta p / 2}\left(y^{\beta}\right)^{p} d y d t .
$$

Now the inner integral is some positive constant; hence, for the double integral to be finite, we require $(\delta p+|\beta| p+n) / 2<-1$, as in (4.8).

In $[16$, chapter $\mathrm{X}$, Theorem 2.1] it is proved that, for $x \in \mathbb{R}, t \geq 0$, and any $\delta>0$,

$$
\left|v_{l}(x, t)\right| \leq(1 / \sqrt{\delta})(t+\delta)^{(l+1) / 2} e^{x^{2} / 4 \delta}(2 l / e)^{l / 2} .
$$

Since the coefficients in (4.1) are positive, we have $\left|v_{l}(x, t)\right| \leq v_{l}(|x|,|t|)$; therefore the above inequality holds also for $t<0$ provided we replace $t$ by $|t|$ on the right. From the product representation (4.2) it follows that, for any multi-index $\beta$ in $\mathbb{N}^{n}$ and any $\delta>0$, and $(x, t) \in \mathbb{R}^{n+1}$,

$$
\left|v_{\beta}(x, t)\right| \leq \delta^{-n / 2}(|t|+\delta)^{(|\beta|+n) / 2} e^{|x|^{2} / 4 \delta}\left(\frac{2}{e}\right)^{|\beta| / 2} \sqrt{\beta^{\beta}} .
$$

In [16, chapter X, Theorem 2.2] it is proved also that, for $x \in \mathbb{R}$ and $t>0$,

$$
\left|v_{l}(x,-t)\right| \leq e^{x^{2} / 8 t}\left(\frac{2 e t}{l}\right)^{l / 2} l ! .
$$

Again from (4.2), it follows that for any multi-index $\beta \in \mathbb{N}^{n}$, and for $x \in \mathbb{R}^{n}$ and $t>0$,

$$
\left|v_{\beta}(x,-t)\right| \leq e^{|x|^{2} / 8 t}(2 e t)^{|\beta| / 2} \beta !\left(\beta^{\beta}\right)^{-1 / 2} .
$$

\section{Expansion of the Heat Kernel in Heat Polynomials}

Widder developed the series representation

$$
K_{1}(x-y, s+t)=\sum_{l=0}^{\infty} \frac{v_{l}(x, t) w_{l}(y, s)}{l ! 2^{l}}
$$

where $w_{l}(y, s)$ is given by

$$
w_{l}(y, s):=s^{-l} K_{1}(y, s) v_{l}(y,-s) .
$$

This representation is valid for $x, y, s, t \in \mathbb{R}$ and $-s<t<s$, and convergence of the series is absolute. (See [16, chapter X, Theorem 3 and Theorem 4.1]). Interchanging $x$ and $y$, and replacing $t$ by $-s$ and $s$ by $t$, we obtain the equivalent formulation

$$
K_{1}(x-y, t-s)=K_{1}(x, t) \sum_{l=0}^{\infty} \frac{v_{l}(x,-t) v_{l}(y,-s)}{l ! 2^{l} t^{l}},
$$

valid for $-t<s<t$. Note that if $t<0$ and $|s|<|t|$, then $t-s<0$ and both sides of (5.1) are 0; therefore, (5.1) is in fact valid for all $s$ and $t$ in $\mathbb{R}$ such that $|s|<|t|$. For the $n$-dimensional heat kernel, (5.1) gives

$$
\begin{aligned}
K_{n}(x-y, t-s)=\prod_{k=1}^{n} K_{1}\left(x_{k}-y_{k}, t-s\right) \\
=\prod_{k=1}^{n} K_{1}\left(x_{k}, t\right) \sum_{l_{k}=0}^{\infty} \frac{v_{l_{k}}\left(x_{k},-t\right) v_{l_{k}}\left(y_{k},-s\right)}{l_{k} ! 2^{l_{k}} t^{l_{k}}} .
\end{aligned}
$$


Since convergence in (5.1) is absolute we may interchange the product with the summation, employing also (4.2) and (4.3), to arrive at

$$
K_{n}(x-y, t-s)=K_{n}(x, t) \sum_{\beta \in \mathbb{N}^{n}} \frac{v_{\beta}(x,-t) v_{\beta}(y,-s)}{\beta ! 2^{|\beta|} t^{|\beta|}},|s|<|t| .
$$

We will require some bounds on the heat kernel. Given any real number $r$, the function $f(\sigma):=(1+\sigma)^{r} e^{-\sigma / 4}$ is bounded on $[0, \infty)$; hence there is a constant $C=C(r)$ such that $f(\sigma) \leq C(r)$. Taking $\sigma=|x|^{2} / t$ yields, for $t>0$,

$$
e^{-|x|^{2} / 4 t} \leq C(r)\left(1+|x|^{2} / t\right)^{-r},
$$

and multiplication by $(4 \pi t)^{-n / 2}$ and some simplification results in

$$
K(x, t) \leq C(r)|t|^{r-n / 2} \sigma(x, t)^{-2 r} .
$$

(The case $t \leq 0$, where $K=0$, is trivial.) Choosing $r=n / 2$ gives

$$
K(x, t) \leq C(n) \sigma(x, t)^{-n} .
$$

Lemma 5.1. Let $\psi=\psi(x, t)$ be a continuous function on $\mathbb{R}^{n+1}$, with $0 \leq \psi \leq 1$ and $\psi(x, t)=0$ when $\sigma(x, t) \leq 1 / 2, \psi(x, t)=1$ when $\sigma(x, t) \geq 1$. For each nonnegative integer $m$ and for $x, y \in \mathbb{R}^{n}$ and $s, t \in \mathbb{R}$, define

$$
K^{(m)}(x, t ; y, s):=K(x-y, t-s)-\psi(x, t) K(x, t) \sum_{|\beta| \leq m} \frac{v_{\beta}(x,-t) v_{\beta}(y,-s)}{\beta ! 2^{|\beta|} t^{|\beta|}} .
$$

Then there exists a constant $C=C(m, n)$ such that

$$
\left|K^{(m)}(x, t ; y, s)\right| \leq C \sigma(x-y, t-s)^{-n} \begin{cases}{\left[\frac{\rho(y, s)}{\rho(x, t)}\right]^{m+n},} & \text { if } \sigma(y, s) \geq \frac{1}{4} \sigma(x, t), \\ {\left[\frac{\rho(y, s)}{\rho(x, t)}\right]^{m+1},} & \text { if } \sigma(y, s) \leq \frac{1}{4} \sigma(x, t) .\end{cases}
$$

Proof. As our first case we suppose that $\sigma(y, s) \geq \sigma(x, t) / 4$. Then

$$
\begin{aligned}
& \sigma(x-y, t-s) \leq \sigma(x, t)+\sigma(y, s) \leq 5 \sigma(y, s), \\
& \rho(x, t) \leq 1+\sigma(x, t) \leq 1+4 \sigma(y, s) \leq 5 \rho(y, s),
\end{aligned}
$$

and, by (5.4) and (5.8),

$$
\begin{aligned}
& K(x-y, t-s) \leq C(n) \sigma(x-y, t-s)^{-n} \\
& \quad \leq C(n) \sigma(x-y, t-s)^{-n} 5^{m+n}\left[\frac{\rho(y, s)}{\rho(x, t)}\right]^{m+n} .
\end{aligned}
$$

By (5.5),

$$
\left|K^{(m)}(x, t ; y, s)\right| \leq K(x-y, t-s)+\psi(x, t) K(x, t) \sum_{|\beta| \leq m} \frac{\left|v_{\beta}(x,-t)\right|\left|v_{\beta}(y,-s)\right|}{\beta ! 2^{|\beta|}|t|^{|\beta|}} .
$$

If $\sigma(x, t) \leq 1 / 2$, then $\psi(x, t)=0$ and (5.6) follows from (5.10) and (5.9); thus we need only consider $\sigma(x, t) \geq 1 / 2$, in which case we have also

$$
\rho(x, t) \leq 1+\sigma(x, t) \leq 3 \sigma(x, t) \leq 3 \rho(x, t) .
$$


From (4.6) and (5.3) with $r=(n / 2)+|\beta|$, we have

$$
K(x, t) \frac{\left|v_{\beta}(x,-t)\right|\left|v_{\beta}(y,-s)\right|}{\beta ! 2^{|\beta|}|t|^{|\beta|}} \leq \frac{C(\beta, n)}{\sigma(x, t)^{n}}\left[\frac{\sigma(y, s)}{\sigma(x, t)}\right]^{|\beta|} .
$$

Then, since $\psi \leq 1$ and $1 \leq 4 \sigma(y, s) / \sigma(x, t)$, and since (5.7) gives also

$$
1 \leq 5 \sigma(y, s) \sigma(x-y, t-s)^{-1}
$$

we find for $|\beta| \leq m$ that

$$
\begin{gathered}
\psi(x, t) K(x, t) \frac{\left|v_{\beta}(x,-t)\right|\left|v_{\beta}(y,-s)\right|}{\beta ! 2^{|\beta|}|t|^{|\beta|}} \leq \frac{C(m, n)}{\sigma(x, t)^{n}}\left[\frac{\sigma(y, s)}{\sigma(x, t)}\right]^{m} \\
\leq \frac{C(m, n)}{\sigma(x-y, t-s)^{n}}\left[\frac{\sigma(y, s)}{\sigma(x, t)}\right]^{m+n} .
\end{gathered}
$$

Substitution of this inequality into (5.10), with the use of $(5.9),(5.11)$, and $\sigma(y, s)<$ $\rho(y, s)$, now yields $(5.6)$ for the case $\sigma(y, s) \geq \sigma(x, t) / 4$.

Now we consider the second case of (5.6), when $\sigma(y, s) \leq \sigma(x, t) / 4$. From $(2.2)$ it follows that

$$
\frac{3}{4} \sigma(x, t) \leq \sigma(x-y, t-s) \leq \frac{5}{4} \sigma(x, t) .
$$

We will consider three subcases, namely,

(a) $\sigma(x, t) \leq 1$,

(b) $\sigma(x, t) \geq 1,|s| \geq \frac{1}{4}|t|$,

(c) $\sigma(x, t) \geq 1,|s| \leq \frac{1}{4}|t|$.

In subcase (a), we have $\rho(x, t) \leq 1+\sigma(x, t) \leq 2 \leq 2 \rho(y, s)$, and so

$$
1 \leq 2 \frac{\rho(y, s)}{\rho(x, t)} \text {. }
$$

Therefore, by (5.4), we have

$$
K(x-y, t-s) \leq C(n) \sigma(x-y, t-s)^{-n} 2^{m+1}\left[\frac{\rho(y, s)}{\rho(x, t)}\right]^{m+1} .
$$

As in the first case, (5.12) holds; then from (5.12)-(5.14) and $\sigma(y, s) / \sigma(x, t) \leq 1 / 4$, $\psi \leq 1$ it follows for $|\beta| \leq m$ that

$$
\begin{gathered}
\psi(x, t) K(x, t) \frac{\left|v_{\beta}(x,-t)\right|\left|v_{\beta}(y,-s)\right|}{\beta ! 2^{|\beta|}|t|^{|\beta|}} \leq \frac{C(\beta, n)}{\sigma(x-y, t-s)^{n}}\left(\frac{1}{4}\right)^{|\beta|} \\
\leq \frac{C(m, n)}{\sigma(x-y, t-s)^{n}}\left[\frac{\rho(y, s)}{\rho(x, t)}\right]^{m+1} .
\end{gathered}
$$

Combining this inequality, (5.15), and (5.10) yields (5.6) for subcase (a).

Next we consider subcase (b). By (5.3) with $r=(m+n+1) / 2$,

$$
K(x-y, t-s) \leq C(m, n) \sigma(x-y, t-s)^{-n}|t-s|^{(m+1) / 2} \sigma(x-y, t-s)^{-m-1} .
$$

In this inequality we use (5.13), along with $\rho(x, t) \leq 1+\sigma(x, t) \leq 2 \sigma(x, t), \sigma(y, s) \leq$ $\rho(y, s)$, and $|t-s| \leq|t|+|s| \leq 5|s| \leq 5 \sigma(y, s)^{2}$, to obtain 


$$
\begin{gathered}
K(x-y, t-s) \leq C(m, n) \sigma(x-y, t-s)^{-n}\left[\frac{\sigma(y, s)}{\sigma(x, t)}\right]^{m+1} \\
\leq C(m, n) \sigma(x-y, t-s)^{-n}\left[\frac{\rho(y, s)}{\rho(x, t)}\right]^{m+1} .
\end{gathered}
$$

Next, (4.6) and (5.3) with $r=(m+n+1+|\beta|) / 2$ give, for $|\beta| \leq m$,

$$
K(x, t) \frac{\left|v_{\beta}(x,-t)\right|\left|v_{\beta}(y,-s)\right|}{\beta ! 2^{|\beta|}|t|^{|\beta|}} \leq C(m, n)|t|^{(m+1-|\beta|) / 2} \sigma(x, t)^{-m-n-1} \sigma(y, s)^{|\beta|} .
$$

We use $|t| \leq 4|s| \leq 4 \sigma(y, s)^{2}$, along with (5.13), and obtain

$$
\begin{gathered}
K(x, t) \frac{\left|v_{\beta}(x,-t)\right|\left|v_{\beta}(y,-s)\right|}{\beta ! 2^{|\beta|}|t|^{|\beta|}} \leq \frac{C(m, n)}{\sigma(x, t)^{n}}\left[\frac{\sigma(y, s)}{\sigma(x, t)}\right]^{m+1} \\
\leq \frac{C(m, n)}{\sigma(x-y, t-s)^{n}}\left[\frac{\rho(y, s)}{\rho(x, t)}\right]^{m+1} .
\end{gathered}
$$

We substitute this inequality and (5.16) into (5.10), again using $\psi \leq 1$, to establish (5.6) for subcase (b).

Finally we consider subcase (c). Since $|s| \leq|t| / 4$, we have (5.2); also, $\psi(x, t)=1$ since $\sigma(x, t) \geq 1$. Therefore (5.5) reduces to

$$
K^{(m)}(x, t ; y, s)=K(x, t) \sum_{|\beta| \geq m+1} \frac{v_{\beta}(x,-t) v_{\beta}(y,-s)}{\beta ! 2^{|\beta|} t^{|\beta|}} .
$$

If $t \leq 0$ then $K(x, t)=0$ and hence $K^{(m)}(x, t ; y, s)=0$; thus we need to verify (5.6) only when $t>0$. We apply (4.11) to $v_{\beta}(x,-t)$ and (4.10) to $v_{\beta}(y,-s)$ with arbitrary $\delta>0$, and obtain from (5.17) the inequality

$$
\left|K^{(m)}(x, t ; y, s)\right| \leq K(x, t) e^{|x|^{2} / 8 t} e^{|y|^{2} / 4 \delta}\left(\frac{|s|+\delta}{\delta}\right)^{n / 2} \sum_{|\beta| \geq m+1}\left(\frac{|s|+\delta}{t}\right)^{|\beta| / 2} .
$$

Now, under our assumptions $\sigma(y, s) \leq \sigma(x, t) / 4$ and $|s| \leq t / 4$, and we can always choose $\delta$ so that we have simultaneously the four inequalities

$$
\begin{aligned}
\frac{|y|^{2}}{4 \delta} \leq 1+\frac{|x|^{2}}{16 t}, & \frac{|s|+\delta}{\delta} \leq 2, \\
& \frac{|s|+\delta}{t} \leq \frac{3}{4}, \quad|s|+\delta \leq 3 \rho(y, s)^{2} .
\end{aligned}
$$

For example, if $|y|^{2} \leq|s|$ we may choose $\delta=2|s|$. If $|y|^{2}>|s|$ and $\rho(y, s)^{2} \leq t / 4$ we may choose $\delta=|y|^{2}+1$. Finally, if $|y|^{2}>|s|$ and $\rho(y, s)^{2} \geq t / 4$ we may choose $\delta=t / 4$. Therefore, we will assume that $\delta$ has been chosen so that (5.19) holds, and proceed to estimate (5.18).

By (2.7) and the first inequality of (5.19),

$$
K(x, t) e^{|x|^{2} / 8 t} e^{|y|^{2} / 4 \delta} \leq(4 \pi t)^{-n / 2} e^{1} e^{-|x|^{2} / 16 t}=C(n) K(x, 4 t) .
$$


Application of (5.3) with $r=(m+n+1) / 2$, along with (5.13) and $\rho(x, t) \leq$ $1+\sigma(x, t) \leq 2 \sigma(x, t)$, then yields

$$
\begin{aligned}
& K(x, t) e^{|x|^{2} / 8 t} e^{|y|^{2} / 4 \delta} \leq C(m, n) \frac{(4 t)^{(m+1) / 2}}{\sigma(x, 4 t)^{m+n+1}} \\
\leq & C(m, n) \frac{t^{(m+1) / 2}}{\sigma(x, t)^{m+n+1}} \leq C(m, n) \frac{t^{(m+1) / 2}}{\sigma(x-y, t-s)^{n}} \rho(x, t)^{-m-1} .
\end{aligned}
$$

The number of multi-indices $\beta$ in $\mathbb{N}^{n}$ with $|\beta|=l$ is

$$
\left(\begin{array}{c}
l+n-1 \\
n-1
\end{array}\right)
$$

therefore, using also (5.19), we may estimate the summation in (5.18) by

$$
\begin{aligned}
\sum_{|\beta| \geq m+1}\left(\frac{|s|+\delta}{t}\right)^{|\beta| / 2} & =\sum_{l=m+1}^{\infty}\left(\frac{|s|+\delta}{t}\right)^{l / 2}\left(\begin{array}{c}
l+n-1 \\
n-1
\end{array}\right) \\
& =\left(\frac{|s|+\delta}{t}\right)^{(m+1) / 2} \sum_{l=0}^{\infty}\left(\frac{|s|+\delta}{t}\right)^{l / 2}\left(\begin{array}{c}
l+m+n \\
n-1
\end{array}\right) \\
& \leq\left(\frac{3 \rho(y, s)^{2}}{t}\right)^{(m+1) / 2} \sum_{l=0}^{\infty}\left(\frac{3}{4}\right)^{l / 2}\left(\begin{array}{c}
l+m+n \\
n-1
\end{array}\right) .
\end{aligned}
$$

The ratio test confirms that the last series converges to some constant, which of course depends on $m$ and $n$; therefore we obtain

$$
\sum_{|\beta| \geq m+1}\left(\frac{|s|+\delta}{t}\right)^{|\beta| / 2} \leq C(m, n) \rho(y, s)^{m+1} t^{-(m+1) / 2} .
$$

We substitute (5.20) and (5.21) into (5.18), using also the second inequality of (5.19), and we have (5.6) for subcase (c).

\section{The Non-ISOmorphism Fredholm Ranges}

We now discuss the range of $\delta$ described in (b) and (c) of Theorem 1.1,

$$
\begin{gathered}
m+n-\frac{n+2}{p}<\delta<1+m+n-\frac{n+2}{p}, \\
-1-m-\frac{n+2}{p}<\delta<-m-\frac{n+2}{p},
\end{gathered}
$$

where $m \in \mathbb{N}$. Note that, in terms of $q,(6.1)$ is equivalent to

$$
-1-m-\frac{n+2}{q}<-\delta-2<-m-\frac{n+2}{q},
$$

while (6.2) is the same as

$$
m+n-\frac{n+2}{q}<-\delta-2<1+m+n-\frac{n+2}{q} .
$$

First we look at the ranges (6.1) and (6.3). By Lemma 4.1 and (6.3), each heat polynomial $v_{\beta}$ with $|\beta| \leq m$ lies in the space $P_{-\delta-2}^{2,1, q}$; thus we have $H_{m} \subset P_{-\delta-2}^{2,1, q} \subset$ $P_{-\delta-2}^{q}$. In particular, $\left\langle f, h^{*}\right\rangle$ is defined if $f \in P_{\delta+2}^{p}$ and $h \in H_{m}$. 
We define operators $\mathcal{G}_{m}, m \in \mathbb{N}$, according to

$$
\mathcal{G}_{m} f(x, t):=\int_{\mathbb{R}^{n+1}} K^{(m)}(x, t ; y, s) f(y, s) d y d s .
$$

Lemma 6.1. Assume $f \in P_{\delta+2}^{p}$ and (6.1) holds; then $\mathcal{G}_{m} f \in P_{\delta}^{p}$ and

$$
\left\|\mathcal{G}_{m} f\right\|_{p ; \rho, \delta} \leq C(m, n, p, \delta)\|f\|_{p ; \rho, \delta+2} .
$$

Moreover, if

$$
\left\langle f, h^{*}\right\rangle=0, \forall h \in H_{m},
$$

then $\mathcal{G}_{m} f \in P_{\delta}^{2,1, p}$ with $\mathcal{H}\left(\mathcal{G}_{m} f\right)=f$, and

$$
\left\|\mathcal{G}_{m} f\right\|_{2,1, p ; \rho, \delta} \leq C(m, n, p, \delta)\|f\|_{p ; \rho, \delta+2} .
$$

Proof. Let $\mathcal{S}$ be the operator of Lemma 2.2. From (5.6) we get the inequality

$$
\begin{aligned}
\left|\mathcal{G}_{m} f(x, t)\right| \leq C(m, n)[ & \rho(x, t)^{-m-n} \mathcal{S}\left(\rho^{m+n}|f|\right)(x, t) \\
& \left.+\rho(x, t)^{-m-1} \mathcal{S}\left(\rho^{m+1}|f|\right)(x, t)\right] .
\end{aligned}
$$

By Lemma 2.2, if $-(n+2) / p<\delta-m-n<n-(n+2) / p$, then

$$
\begin{aligned}
& \left\|\rho^{-(m+n)} \mathcal{S}\left(\rho^{m+n}|f|\right)\right\|_{p ; \rho, \delta}=\left\|\mathcal{S}\left(\rho^{m+n}|f|\right)\right\|_{p ; \rho, \delta-m-n} \\
& \leq C(m, n, p, \delta)\left\|\rho^{m+n} f\right\|_{p ; \rho, \delta+2-m-n}=C(m, n, p, \delta)\|f\|_{p ; \rho, \delta+2},
\end{aligned}
$$

and similarly, if $-(n+2) / p<\delta-m-1<n-(n+2) / p$, then

$$
\left\|\rho^{-(m+1)} \mathcal{S}\left(\rho^{m+1}|f|\right)\right\|_{p ; \rho, \delta} \leq C(m, n, p, \delta)\|f\|_{p ; \rho, \delta+2} .
$$

Thus (6.6) is valid for $\delta$ in the range (6.1), as determined by our two conditions on $\delta$.

Now suppose that also (6.7) holds. From the representations (5.5) and (6.5) we see that $\mathcal{G}_{m} f=\mathcal{K} f$, since $v_{\beta} \in H_{m}$ when $|\beta| \leq m$. Also, $(6.1)$ gives $-(n+2) / p<$ $\delta-m-n<1-(n+2) / p$, so Theorem 2.4 says that $\mathcal{G}_{m} f \in P_{\delta-m-n}^{2,1, p}$, with $\mathcal{H}\left(\mathcal{G}_{m} f\right)=f$. Now Lemma 2.3 gives

$$
\left\|\mathcal{G}_{m} f\right\|_{2,1, p ; \rho, \delta} \leq C(n, p, \delta)\left[\left\|\mathcal{G}_{m} f\right\|_{p ; \rho, \delta}+\|f\|_{p ; \rho, \delta+2}\right]
$$

into which we substitute (6.6) to obtain (6.8).

Theorem 6.2. If (6.1) holds, then the mapping $\mathcal{H}: P_{\delta}^{2,1, p} \rightarrow P_{\delta+2}^{p}$ is one-to-one, with range

$$
R_{m, p, \delta}:=\left\{f \in P_{\delta+2}^{p}:\left\langle f, h^{*}\right\rangle=0, \forall h \in H_{m}\right\} .
$$

Proof. By Theorem 3.2, $\mathcal{H}$ is one-to-one. Lemma 6.1 shows that if $f \in R_{m, p, \delta}$, then $\mathcal{G}_{m} f \in P_{\delta}^{2,1, p}$ with $\mathcal{H}\left(\mathcal{G}_{m} f\right)=f$; thus $R_{m, p, \delta} \subset($ range $\mathcal{H})$. On the other hand, if $f$ is in the range of $\mathcal{H}$, say $f=\mathcal{H} u$ for some $u$ in $P_{\delta}^{2,1, p}$, then we use Lemma 3.1 to observe that, for all $h \in H_{m} \subset P_{-\delta-2}^{2,1, q}$,

$$
\left\langle f, h^{*}\right\rangle=\left\langle\mathcal{H} u, h^{*}\right\rangle=\left\langle u^{*}, \mathcal{H} h\right\rangle=\left\langle u^{*}, 0\right\rangle=0 .
$$

Therefore (range $\mathcal{H}) \subset R_{m, p, \delta}$.

Corollary 6.3. If (6.1) holds and $u \in P_{\delta}^{2,1, p}$, then $u=\mathcal{G}_{m}(\mathcal{H} u)$.

Proof. We have $\mathcal{H} u \in P_{\delta+2}^{p}$ and, by Theorem $6.2,\left\langle\mathcal{H} u, h^{*}\right\rangle=0$ for all $h \in H_{m}$. By Lemma 6.1, $\mathcal{G}_{m}(\mathcal{H} u) \in P_{\delta}^{2,1, p}$ with $\mathcal{H}\left(\mathcal{G}_{m}(\mathcal{H} u)\right)=\mathcal{H} u$. Since $\mathcal{H}: P_{\delta}^{2,1, p} \rightarrow P_{\delta+2}^{p}$ is one-to-one, $u=\mathcal{H}\left(\mathcal{G}_{m} u\right)$. 
Next we investigate the ranges (6.2) and (6.4), in which case Lemma 4.1 implies that $H_{m} \subset P_{\delta}^{2,1, p}$.

Theorem 6.4. If (6.2) holds, then the mapping $\mathcal{H}: P_{\delta}^{2,1, p} \rightarrow P_{\delta+2}^{p}$ is onto, with kernel $H_{m}$.

Proof. For fixed $f \in P_{\delta+2}^{p}$, let $\mathcal{L}$ be the mapping defined on $P_{-\delta}^{q}$ according to

$$
\mathcal{L}(v):=\left\langle f,\left(\mathcal{G}_{m} v\right)^{*}\right\rangle .
$$

Since (6.4) holds, we may use (2.4) and Lemma (6.1) to obtain the bound

$$
|\mathcal{L}(v)| \leq\|f\|_{p ; \rho, \delta+2}\left\|\mathcal{G}_{m} v\right\|_{q ; \rho,-\delta-2} \leq\|f\|_{p ; \rho, \delta+2} C(m, n, p, \delta)\|v\|_{q ; \rho,-\delta} ;
$$

therefore, there exists a function $u$ in $P_{\delta}^{p}$ such that, for all $v \in P_{-\delta}^{q}$,

$$
\left\langle u^{*}, v\right\rangle=\mathcal{L}(v)=\left\langle f,\left(\mathcal{G}_{m} v\right)^{*}\right\rangle .
$$

We take $v=\mathcal{H} \phi$, where $\phi \in C_{0}^{\infty}\left(\mathbb{R}^{n+1}\right)$; then $\phi=\mathcal{G}_{m}(\mathcal{H} \phi)$ by Corollary 6.3 , and we have $\left\langle u^{*}, \mathcal{H} \phi\right\rangle=\left\langle f, \phi^{*}\right\rangle$. Thus $\mathcal{H} u=f$ by (2.6), and $u \in P_{\delta}^{2,1, p}$ by Corollary 2.5 ; hence $\mathcal{H}$ is onto.

It is clear that the kernel of $\mathcal{H}$ contains $H_{m}$. We suppose that $u$ is in $P_{\delta}^{2,1, p}$ with $\mathcal{H} u=0$, and show that $u \in H_{m}$. By Lemma 3.1, if $v \in P_{-\delta-2}^{2,1, q}$ then

$$
\left\langle u,(\mathcal{H} v)^{*}\right\rangle=\left\langle u^{*}, \mathcal{H} v\right\rangle=\left\langle\mathcal{H} u, v^{*}\right\rangle=\left\langle 0, v^{*}\right\rangle=0 .
$$

Since (6.4) holds, Theorem 6.2 states that

$$
\begin{aligned}
& \left\{(\mathcal{H} v)^{*}: v \in P_{-\delta-2}^{2,1, q}\right\}=\left\{f^{*} \in P_{-\delta}^{q}:\left\langle f, h^{*}\right\rangle=0, \forall h \in H_{m}\right\} \\
& =\left\{g \in P_{-\delta}^{q}:\langle g, h\rangle=0, \forall h \in H_{m}\right\}=\left(H_{m}\right)^{\perp},
\end{aligned}
$$

where $P_{\delta}^{p}$ and $P_{-\delta}^{q}$ are viewed as dual spaces of one another. We now have $u \in P_{\delta}^{p}$ and $u \perp\left(H_{m}\right)^{\perp}$. But $\left(H_{m}^{\perp}\right)^{\perp}=H_{m}$, since $H_{m}$ is finite dimensional and hence closed; thus $u \in H_{m}$.

Corollary 6.5. In cases (a), (b), (c) of Theorem 1, the mapping ( $\dagger)$ is Fredholm, with Fredholm index

$$
0, \quad-\left(\begin{array}{c}
m+n \\
n
\end{array}\right), \quad\left(\begin{array}{c}
m+n \\
n
\end{array}\right)
$$

respectively.

Proof. We have ind $\mathcal{H}=$ nul $\mathcal{H}$ - def $\mathcal{H}$, where nul $\mathcal{H}=\operatorname{def} \mathcal{H}=0$ in (a), nul $\mathcal{H}=0$ and $\operatorname{def} \mathcal{H}=\operatorname{codim} \mathcal{H}_{m}^{\perp}=\operatorname{dim} \mathcal{H}_{m}$ in (b), nul $\mathcal{H}=\operatorname{dim} \mathcal{H}_{m}$ and def $\mathcal{H}=0$ in (c). (Recall formula (4.5).)

\section{The Non-Fredholm Values}

We now establish parts (d) and (e) of Theorem 1.1.

Theorem 7.1. If $\delta=-m-(n+2) / p$ for some $m \in \mathbb{N}$, then the mapping

$$
\mathcal{H}: P_{\delta}^{2,1, p} \rightarrow P_{\delta+2}^{p}
$$

has kernel $H_{m-1}$, and the range of $\mathcal{H}$ is not closed. 
Proof. By Theorem 6.4, if $0<\epsilon<1$, then the mapping between the larger spaces

$$
\mathcal{H}: P_{\delta-\epsilon}^{2,1, p} \rightarrow P_{\delta-\epsilon+2}^{p}
$$

has kernel $H_{m}$; hence the mapping $(\dagger)$ has kernel $H_{m} \cap P_{\delta}^{2,1, p}$. By Lemma 4.1, $v_{\beta} \in P_{\delta}^{2,1, p}$ iff $\delta<-|\beta|-(n+2) / p$; thus $H_{m-1}$ is the kernel of $(\dagger)$. (When $m=0$ we define $H_{-1}:=\{0\}=H_{0} \cap P_{\delta}^{2,1, p}$; in this case, $(\dagger)$ is one-to-one.)

Let $v_{\beta}$ be any heat polynomial with $|\beta|=m$; then $v_{\beta} \in H_{m}-H_{m-1}$. Let $\psi=\psi(x, t)$ be any function in $C_{0}^{\infty}\left(\mathbb{R}^{n+1}\right)$ such that $0 \leq \psi \leq 1, \psi=1$ whenever $\sigma(x, t) \leq 1, \psi \equiv 0$ whenever $\sigma(x, t) \geq 2$, and for constants $\lambda>0$ further define

$$
u_{\lambda}(x, t):=v_{\beta}(x, t) \psi\left(x / \lambda, t / \lambda^{2}\right) .
$$

Then $u_{\lambda} \in C_{0}^{\infty}\left(\mathbb{R}^{n+1}\right),\left|u_{\lambda}\right| \leq\left|v_{\beta}\right|$, and $u_{\lambda} \equiv v_{\beta}$ whenever $\sigma(x, t) \leq \lambda, u_{\lambda} \equiv 0$ whenever $\sigma(x, t) \geq 2 \lambda$. In particular, $u_{\lambda} \in P_{\delta}^{2,1, p}$. In order to show that the range of $\mathcal{H}$ is not closed in $P_{\delta+2}^{p}$, it is sufficient to demonstrate that

$$
\lim _{\lambda \rightarrow \infty} \frac{\left\|\mathcal{H} u_{\lambda}\right\|_{p ; \rho, \delta+2}}{\operatorname{dist}\left(u_{\lambda}, \operatorname{ker} \mathcal{H}\right)}=0 .
$$

(See [7, pg. 231, Theorem 5.2].) We will show that the numerator in (7.2) is bounded for $\lambda \geq 1$, while the denominator tends to infinity as $\lambda \rightarrow \infty$.

We differentiate (7.1) and use $\mathcal{H} v_{\beta}=0$ to obtain

$$
\mathcal{H} u_{\lambda}(x, t)=-2 \lambda^{-1} \nabla v_{\beta}(x, t) \cdot \nabla \psi\left(x / \lambda, t / \lambda^{2}\right)+\lambda^{-2} v_{\beta}(x, t) \mathcal{H} \psi\left(x / \lambda, t / \lambda^{2}\right),
$$

and then use (4.6) and (4.7) with the observations that $|\nabla \psi|,|\mathcal{H} \psi| \leq C(\psi), \nabla \psi \equiv$ $\mathcal{H} \psi \equiv 0$ except where $\lambda \leq \sigma(x, t) \leq 2 \lambda$, to conclude that

$$
\left|\mathcal{H} u_{\lambda}(x, t)\right| \leq \begin{cases}C(m, n, \psi)\left[\lambda^{-1} \sigma(x, t)^{m-1}+\lambda^{-2} \sigma(x, t)^{m}\right], & \text { if } \lambda \leq \sigma(x, t) \leq 2 \lambda, \\ 0, & \text { otherwise }\end{cases}
$$

Now we investigate the integrals, for $l=0$ or $l=1$ and $\lambda \geq 1$,

$$
J(l):=\int_{\lambda \leq \sigma(x, t) \leq 2 \lambda} \rho(x, t)^{(\delta+2) p}\left[\lambda^{l-2} \sigma(x, t)^{m-l}\right]^{p} d x d t .
$$

The change of variables, from $(x, t) \in \mathbb{R}^{n} \times \mathbb{R}$ to $y \in \mathbb{R}^{n+1}$, described by

$$
\begin{gathered}
y=(x, t / \sqrt{|t|}), \quad d x d t=2 \sqrt{|t|} d y \leq 2|y| d y, \\
\sigma(x, t)=|y|, \quad \rho(x, t)=\left(1+|y|^{2}\right)^{1 / 2},
\end{gathered}
$$

yields

$$
J(l) \leq 2 \lambda^{(l-2) p} \int_{\lambda \leq|y| \leq 2 \lambda}\left(1+|y|^{2}\right)^{(\delta+2) p / 2}|y|^{(m-l) p}|y| d y \leq C(m, n, p, \delta) .
$$

Combination of this estimate with (7.3) shows that the numerator of (7.2) is bounded, independently of $\lambda$ when $\lambda \geq 1$.

Since $v_{\beta} \notin P_{\delta}^{p}$ by Lemma 4.1, the norms $\left\|u_{\lambda}\right\|_{p ; \rho, \delta}$ approach infinity as $\lambda \rightarrow \infty$. We show that the denominator of (7.2) tends to infinity by showing that, when $\lambda$ is sufficiently large,

$$
\left\|u_{\lambda}-w\right\|_{p ; \rho, \delta} \geq \frac{1}{2}\left\|u_{\lambda}\right\|_{p ; \rho, \delta}, \text { for } w \in H_{m-1} .
$$


Note that when $m=0$, then $w=0$ and we are done; thus we may assume $m \geq 1$. We may also restrict our attention to functions $w$ such that

$$
\|w\|_{p ; \rho, \delta} \leq \frac{3}{2}\left\|u_{\lambda}\right\|_{p ; \rho, \delta},
$$

as otherwise (7.5) follows from the triangle inequality.

For $w \in H_{m-1}$,

$$
\left(\left\|u_{\lambda}-w\right\|_{p ; \rho, \delta}\right)^{p}=\int_{\mathbb{R}^{n+1}} \rho(x, t)^{\delta p}\left|v_{\beta}(x, t) \psi\left(x / \lambda, t / \lambda^{2}\right)-w(x, t)\right|^{p} d x d t .
$$

We suppose $0<\epsilon<1$, and in the integral substitute $x=y / \epsilon, t=s / \epsilon^{2}$, with $d x d t=\epsilon^{-n-2} d y d s$, and use the property (4.4) on $v_{\beta}$ to obtain

$$
\begin{aligned}
& \left(\left\|u_{\lambda}-w\right\|_{p ; \rho, \delta}\right)^{p} \\
& =\epsilon^{-m p-n-2} \int_{\mathbb{R}^{n+1}} \rho\left(y / \epsilon, s / \epsilon^{2}\right)^{\delta p}\left|v_{\beta}(y, s) \psi\left(y / \lambda \epsilon, s / \epsilon^{2} \lambda^{2}\right)-\epsilon^{m} w\left(y / \epsilon, s / \epsilon^{2}\right)\right|^{p} d y d s .
\end{aligned}
$$

But $\delta p+m p+n+2=0$ implies $\delta p<0 ;$ therefore,

$$
\rho\left(y / \epsilon, s / \epsilon^{2}\right)^{\delta p}=\left(1+\frac{|y|^{2}}{\epsilon^{2}}+\frac{|s|}{\epsilon^{2}}\right)^{\delta p / 2} \geq \epsilon^{-\delta p} \rho(y, s)^{\delta p} .
$$

Setting

$$
w_{\epsilon}(y, s):=\epsilon^{m} w\left(y / \epsilon, s / \epsilon^{2}\right)
$$

we find that

$$
\left(\left\|u_{\lambda}-w\right\|_{p ; \rho, \delta}\right)^{p} \geq \int_{\mathbb{R}^{n+1}} \rho(y, s)^{\delta p}\left|v_{\beta}(y, s) \psi\left(y / \lambda \epsilon, s / \lambda^{2} \epsilon^{2}\right)-w_{\epsilon}(y, s)\right|^{p} d y d s,
$$

and hence

$$
\left\|u_{\lambda}-w\right\|_{p ; \rho, \delta} \geq\left\|u_{\lambda \epsilon}-w_{\epsilon}\right\|_{p ; \rho, \delta}
$$

Let $V$ be the vector space of all real polynomials $w$ in $\mathbb{R}^{n+1}$ of the form

$$
w(x, t)=\sum_{|\alpha| \leq m-1,2 \gamma \leq \alpha} a_{\alpha, \gamma} x^{\alpha-2 \gamma} t^{|\gamma|} .
$$

We have $H_{m-1} \subset V$, since each polynomial in $H_{m-1}$ is a linear combination of heat polynomials of the form (4.3), with $|\beta| \leq m-1$. In the proof of Lemma 4.1 it was shown that, for polynomials $w$ of this form, $\|w\|_{p ; \rho, \delta}<\infty$ provided that $\delta<-(m-1)-(n+2) / p$; thus $V \subset P_{\delta}^{p}$. On $V$ we may also define a norm based on the coefficients in (7.9),

$$
\|w\|_{\text {coef }}:=\sup _{\alpha, \gamma}\left|a_{\alpha, \gamma}\right|
$$

Since any two norms on a finite dimensional space are equivalent, there is a constant $M>0$ such that, for all $w \in V$,

$$
\frac{1}{M}\|w\|_{\text {coef }} \leq\|w\|_{p ; \rho, \delta} \leq M\|w\|_{\text {coef }}
$$

Now, if $w$ is given by (7.9) and $w_{\epsilon}$ by (7.7), then

$$
w_{\epsilon}(x, t)=\sum_{|\alpha| \leq m-1,2 \gamma \leq \alpha}\left(\epsilon^{m-|\alpha|} a_{\alpha, \gamma}\right) x^{\alpha-2 \gamma} t^{|\gamma|},
$$


and $\left\|w_{\epsilon}\right\|_{\text {coef }} \leq \epsilon\|w\|_{\text {coef }}$; therefore,

$$
\left\|w_{\epsilon}\right\|_{p ; \rho, \delta} \leq M\left\|w_{\epsilon}\right\|_{\text {coef }} \leq M \epsilon\|w\|_{\text {coef }} \leq \epsilon M^{2}\|w\|_{p ; \rho, \delta} .
$$

From (7.8), the triangle inequality, (7.11) and (7.6) it follows that

$$
\begin{aligned}
\left\|u_{\lambda}-w\right\|_{p ; \rho, \delta} & \geq\left\|u_{\lambda \epsilon}\right\|_{p ; \rho, \delta}-\left\|w_{\epsilon}\right\|_{p ; \rho, \delta} \geq\left\|u_{\lambda \epsilon}\right\|_{p ; \rho, \delta}-\epsilon M^{2}\|w\|_{p ; \rho, \delta} \\
& \geq\left\|u_{\lambda}\right\|_{p ; \rho, \delta}-\left\|u_{\lambda}-u_{\lambda \epsilon}\right\|_{p ; \rho, \delta}-\frac{3}{2} \epsilon M^{2}\left\|u_{\lambda}\right\|_{p ; \rho, \delta} .
\end{aligned}
$$

We choose $\epsilon$ so that $3 \epsilon M^{2} / 2=1 / 3$; then

$$
\left\|u_{\lambda}-w\right\|_{p ; \rho, \delta} \geq \frac{2}{3}\left\|u_{\lambda}\right\|_{p ; \rho, \delta}-\left\|u_{\lambda}-u_{\lambda \epsilon}\right\|_{p ; \rho, \delta} .
$$

Since $\delta p<0$, we have $\rho^{\delta p} \leq \sigma^{\delta p}$, and the estimate

$$
\left(\left\|u_{\lambda}-u_{\lambda \epsilon}\right\|_{p ; \rho, \delta}\right)^{p}=\int_{\mathbb{R}^{n+1}} \rho^{\delta p}\left|u_{\lambda}-u_{\lambda \epsilon}\right|^{p} d x d t \leq \int_{\lambda \epsilon \leq \sigma(x, t) \leq 2 \lambda} \sigma^{\delta p}\left|v_{\beta}\right|^{p} d x d t .
$$

We apply (4.6), and again make the change of variables (7.4), to obtain

$$
\left(\left\|u_{\lambda}-u_{\lambda \epsilon}\right\|_{p ; \rho, \delta}\right)^{p} \leq C(m, n) \int_{\lambda \epsilon \leq|y| \leq 2 \lambda}|y|^{(\delta p+m p+1)} d y=C(m, n) \log \frac{2}{\epsilon} .
$$

Therefore, since $\left\|u_{\lambda}\right\|_{p ; \rho, \delta} \rightarrow \infty$, we may choose $\lambda$ sufficiently large that

$$
\left\|u_{\lambda}-u_{\lambda \epsilon}\right\|_{p ; \rho, \delta} \leq \frac{1}{6}\left\|u_{\lambda}\right\|_{p ; \rho, \delta}
$$

then (7.12) gives (7.5).

Theorem 7.2. If $\delta=m+n-(n+2) / p$ for some $m \in \mathbb{N}$, then the mapping

$$
\mathcal{H}: P_{\delta}^{2,1, p} \rightarrow P_{\delta+2}^{p}
$$

is one-to-one, and the range of $\mathcal{H}$ is not closed.

Proof. By Theorem 3.2, $\mathcal{H}$ is one-to-one. Since $-\delta-2=-m-(n+2) / q$, the pair $(-\delta-2, q)$ satisfies the hypothesis of Theorem 7.1. We assume the range of $\mathcal{H}$ in $(\dagger)$ is closed, and show that the mapping $\mathcal{H}: P_{-\delta-2}^{2,1, q} \rightarrow P_{-\delta}^{q}$ is onto, thereby contradicting Theorem 7.1.

If $(\dagger)$ is one-to-one with closed range, then $\mathcal{H}$ is bounded below; that is, there exists $\epsilon>0$ such that, for all $w$ in $P_{\delta}^{2,1, p}$,

$$
\|\mathcal{H} w\|_{p ; \rho, \delta+2} \geq \epsilon\|w\|_{2,1, p ; \rho, \delta} \geq \epsilon\|w\|_{p ; \rho, \delta} .
$$

Let $f \in P_{-\delta}^{q}$. The linear mapping $\mathcal{L}:$ range $\mathcal{H} \rightarrow \mathbb{R}$, defined by

$$
\mathcal{L}(v):=\left\langle f,\left(\mathcal{H}^{-1} v\right)^{*}\right\rangle,
$$

is bounded, as confirmed by

$$
|\mathcal{L}(v)| \leq\|f\|_{q ; \rho,-\delta}\left\|\mathcal{H}^{-1} v\right\|_{p ; \rho, \delta} \leq\|f\|_{q ; \rho,-\delta} \frac{1}{\epsilon}\|v\|_{p ; \rho, \delta+2} .
$$

By the Hahn-Banach Theorem, there exists $u \in P_{-\delta-2}^{q}$ such that, for all $w$ in the range of $\mathcal{H},\left\langle u^{*}, v\right\rangle=\mathcal{L}(v)=\left\langle f,\left(\mathcal{H}^{-1} v\right)^{*}\right\rangle$. Taking a particular $v=\mathcal{H} \phi$, where $\phi \in C_{0}^{\infty}\left(\mathbb{R}^{n+1}\right)$, gives $\left\langle u^{*}, \mathcal{H} \phi\right\rangle=\left\langle f, \phi^{*}\right\rangle$. By (2.6) and Corollary 2.5, $\mathcal{H} u=f$ and $u \in P_{-\delta-2}^{2,1, q}$; hence, $\mathcal{H}: P_{-\delta-2}^{2,1, q} \rightarrow P_{-\delta}^{q}$ is onto. 


\section{Proofs of Technical Lemmas}

In this section we supply the proofs of Lemmas 2.1 and 2.2

Proof of Lemma 2.1. Let $u$ be a function in $P_{\delta}^{m, k, p}$, and suppose $\epsilon>0$; we show that there exists a function $\phi$ in $C_{0}^{\infty}\left(\mathbb{R}^{n+1}\right)$ with $\|u-\phi\|_{2,1, p ; \rho, \delta}<\epsilon$. Let $\psi$ be a real valued function in $C_{0}^{\infty}\left(\mathbb{R}^{n+1}\right)$ such that $\psi \equiv 1$ in the region where $\sigma(x, t) \leq 1$, $\psi \equiv 0$ wherever $\sigma(x, t) \geq 2$, and $0 \leq \psi \leq 1$ otherwise. Let $R$ be a large constant, say with $R \geq 1$, and set

$$
\psi_{R}(x, t):=\psi\left(x / R, t / R^{2}\right) .
$$

Then, $\psi_{R} \in C_{0}^{\infty}\left(\mathbb{R}^{n+1}\right), 0 \leq \psi_{R} \leq 1, \psi_{R} \equiv 0$ where $\sigma(x, t) \leq R, \psi_{R} \equiv 1$ where $\sigma(x, t) \geq 2 R$, and for multi-indices $\alpha$ and nonnegative integers $k$,

$$
D_{x}^{\alpha} \psi_{R}(x, t)=R^{-|\alpha|} D_{x}^{\alpha} \psi\left(x / R, t / R^{2}\right), \quad D_{t}^{k} \psi_{R}(x, t)=R^{-2 k} D_{t}^{k} \psi\left(x / R, t / R^{2}\right) .
$$

It follows that, for some positive constants $C(\psi, \alpha)$ and $C(\psi, k)$,

$$
\left|D_{x}^{\alpha} \psi_{R}(x, t)\right| \leq C(\psi, \alpha) R^{-|\alpha|}, \quad D_{t}^{k} \psi_{R}(x, t) \mid \leq C(\psi, k) R^{-2 k} .
$$

Let $v$ be the function $v:=\psi_{R} u$. Then $v \in P_{\delta}^{m, k, p}, v=u$ whenever $\sigma(x, t) \leq R$, $v=0$ where $\sigma(x, t) \geq 2 R$. We show that $\|v-u\|_{m, k, p ; \rho, \delta}<\epsilon / 2$ provided that $R$ is sufficiently large.

Let $\Omega_{R}$ be the region where $\sigma(x, t) \geq R$, and $\Omega_{R, 2 R}$ the region where $R \leq$ $\sigma(x, t) \leq 2 R$; then $v-u \equiv 0$ except in $\Omega_{R}$, and the triangle inequality gives

$$
\left\|\rho^{\delta+|\alpha|} D_{x}^{\alpha}(v-u)\right\|_{p} \leq\left\|\rho^{\delta+|\alpha|} D_{x}^{\alpha} v\right\|_{p ; \Omega_{R, 2 R}}+\left\|\rho^{\delta+|\alpha|} D_{x}^{\alpha} u\right\|_{p ; \Omega_{R}} .
$$

From (8.1) and the fact that $R \leq \rho \leq 3 R$ in $\Omega_{R, 2 R}$ it follows that

$$
\begin{aligned}
\left\|\rho^{\delta+|\alpha|} D_{x}^{\alpha} v\right\|_{p ; \Omega_{R, 2 R}} & =\left\|\rho^{\delta+|\alpha|} \sum_{\beta \leq \alpha}\left(\begin{array}{c}
\alpha \\
\beta
\end{array}\right) D_{x}^{\beta} u D_{x}^{\alpha-\beta} \psi_{R}\right\|_{p ; \Omega_{R, 2 R}} \\
& \leq C(\psi, \alpha)\left\|\rho^{\delta+|\alpha|} \sum_{\beta \leq \alpha} R^{|\beta|-|\alpha|} D_{x}^{\beta} u\right\|_{p ; \Omega_{R, 2 R}} \\
& \leq C(\psi, \alpha) \sum_{\beta \leq \alpha}\left\|\rho^{\delta+|\beta|} D_{x}^{\beta} u\right\|_{p ; \Omega_{R}}
\end{aligned}
$$

and then from (8.2) that

$$
\left\|\rho^{\delta+|\alpha|} D_{x}^{\alpha}(v-u)\right\|_{p} \leq C(\psi, \alpha) \sum_{\beta \leq \alpha}\left\|\rho^{\delta+|\beta|} D_{x}^{\beta} u\right\|_{p ; \Omega_{R}} .
$$

With similar calculations we can show that

$$
\left\|\rho^{\delta+2 k} D_{t}^{k}(v-u)\right\|_{p} \leq C(\psi, k) \sum_{j=0}^{k}\left\|\rho^{\delta+2 j} D_{t}^{j} u\right\|_{p ; \Omega_{R}} .
$$

Since $\|u\|_{m, k, p ; \rho, \delta}$ is finite, as $R \rightarrow \infty$ we have for $|\beta| \leq m$ and $j \leq k$ that

$$
\left\|\rho^{\delta+|\beta|} D_{x}^{\beta} u\right\|_{p ; \Omega_{R}} \rightarrow 0, \quad\left\|\rho^{\delta+2 j} D_{t}^{j} u\right\|_{p ; \Omega_{R}} \rightarrow 0 ;
$$

therefore, (8.3) and (8.4) show that we can make $\|v-u\|_{m, k, p ; \rho, \delta}<\epsilon / 2$ by choosing $R$ large enough.

Now we use a standard argument involving mollifiers to show that there is a function $\phi$ in $C_{0}^{\infty}\left(\mathbb{R}^{n+1}\right)$ with $\|\phi-v\|_{m, k, p ; \rho, \delta}<\epsilon / 2$, thereby completing the proof of the theorem. (See chapter 7 of [4], for example, for relevant theory on mollifiers.) 
Let $\eta$ be a nonnegative function in $C_{0}^{\infty}\left(\mathbb{R}^{n+1}\right)$ vanishing outside the unit ball $\mathbb{B}_{1}(0)$, with $\int \eta d x=1$. For $s>0$ set $\eta_{s}(x, t):=s^{-n-1} \eta(x / s, t / s)$, and let $v_{s}$ be the convolution $v_{s}:=\eta_{s} * v$. The support of $v$ lies in some ball $\mathbb{B}_{r}(0)$ with $r \geq 1$, and it follows that $v_{s} \in C_{0}^{\infty}\left(\mathbb{R}^{n+1}\right)$ with the support of $v_{s}$ in $\mathbb{B}_{2 r}(0)$ if $s<r$. For $|\alpha| \leq m$ we have $D_{x}^{\alpha}\left(v_{s}\right)=\left(D_{x}^{\alpha} v\right)_{s}$ and $\left\|\left(D_{x}^{\alpha} v\right)_{s}-D_{x}^{\alpha} v\right\|_{p} \rightarrow 0$ as $s \rightarrow 0$; and likewise for $j \leq k$ we have $D_{t}^{j}\left(v_{s}\right)=\left(D_{t}^{j} v\right)_{s}$ and $\left\|\left(D_{t}^{j} v\right)_{s}-D_{t}^{j} v\right\|_{p} \rightarrow 0$. We can check that $\rho(x, t) \leq 3 r$ in $\mathbb{B}_{2 r}(0)$, and hence

$$
\begin{gathered}
\left\|\rho^{\delta+|\alpha|} D_{x}^{\alpha}\left(v_{s}-v\right)\right\|_{p} \leq(3 r)^{\delta+|\alpha|}\left\|\left(D_{x}^{\alpha} v\right)_{s}-D_{x}^{\alpha} v\right\|_{p} \rightarrow 0, \\
\left\|\rho^{\delta+2 j} D_{t}^{j}\left(v_{s}-v\right)\right\|_{p} \leq(3 r)^{\delta+2 j}\left\|\left(D_{t}^{j} v\right)_{s}-D_{t}^{j} v\right\|_{p} \rightarrow 0 .
\end{gathered}
$$

Setting $\phi \equiv v_{s}$, we conclude that $\|\phi-v\|_{m, k, p ; \rho, \delta}<\epsilon / 2$ if $s$ is small enough.

Before proving Lemma 2.2, we require another lemma:

Lemma 8.1. Given real numbers $\xi$ and $\eta$, and $(x, t) \in \mathbb{R}^{n+1}$, define

$$
J(x, t, n, \xi, \eta):=\int_{\mathbb{R}^{n+1}} \sigma(x-y, t-s)^{\xi} \rho(y, s)^{\eta} d y d s .
$$

If

$$
n+2+\xi>0, n+2+\eta>0, n+2+\xi+\eta<0,
$$

then

$$
J(x, t, n, \xi, \eta) \leq C(n, \xi, \eta) \rho(x, t)^{n+2+\xi+\eta} .
$$

Proof. We divide $\mathbb{R}^{n+1}$ into three regions

$$
\begin{aligned}
& R_{1}:=\left\{(y, s): \sigma(x-y, t-s) \leq \frac{1}{2} \rho(x, t)\right\}, \\
& R_{2}:=\left\{(y, s): \frac{1}{2} \rho(x, t)<\sigma(x-y, t-s)<2 \rho(x, t)\right\}, \\
& R_{3}:=\{(y, s): 2 \rho(x, t) \leq \sigma(x-y, t-s)\},
\end{aligned}
$$

and write $J(x, t, n, \xi, \eta)=J_{1}+J_{2}+J_{3}$, where

$$
J_{i}:=\int_{R_{i}} \sigma(x-y, t-s)^{\xi} \rho(y, s)^{\eta} d y d s, i=1,2,3 .
$$

It is sufficient to show that, for each $i,(8.7)$ holds with $J$ replaced by $J_{i}$. Note that (8.6) implies that $\xi<0$ and $\eta<0$.

In the region $R_{1}$ we have the conservative estimates

$$
\rho(x, t) \leq 1+\sigma(x, t) \leq 1+\sigma(x-y, t-s)+\sigma(y, s) \leq \frac{1}{2} \rho(x, t)+2 \rho(y, s),
$$

and thereby $\rho(x, t) \leq 4 \rho(y, s)$. Since $\eta<0$, we may substitute $\rho(x, t)$ for $\rho(y, s)$ into (8.8), and then set $z=y-x, r=s-t$ to obtain

$$
J_{1} \leq C(\eta) \rho(x, t)^{\eta} \int_{\sigma(z, r) \leq \rho(x, t)} \sigma(z, r)^{\xi} d z d r .
$$

In the last integral we substitute $w=(z, r / \sqrt{|r|})$, with $\sigma(z, r)=|w|$ and $d z d r=$ $2 \sqrt{|r|} d w \leq 2|w| d w$, using also $n+2+\xi>0$, and arrive at

$$
\begin{aligned}
J_{1} & \leq C(\eta) \rho(x, t)^{\eta} \int_{|w| \leq \rho(x, t)} 2|w|^{\xi+1} d w \\
& =C(\eta) \rho(x, t)^{\eta} C(n, \xi) \rho(x, t)^{n+2+\xi}=C(n, \xi, \eta) \rho(x, t)^{n+2+\xi+\eta} .
\end{aligned}
$$


In the region $R_{2}$, besides $\frac{1}{2} \rho(x, t) \leq \sigma(x-y, t-s) \leq 2 \rho(x, t)$ we have also

$$
\sigma(y, s) \leq \sigma(x-y, t-s)+\sigma(x, t) \leq 2 \rho(x, t)+\rho(x, t)=3 \rho(x, t) ;
$$

therefore, from (8.8) it follows that

$$
J_{2} \leq C(\xi) \rho(x, t)^{\xi} \int_{\sigma(y, s) \leq 3 \rho(x, t)} \rho(y, s)^{\eta} d y d s .
$$

As in $J_{1}$ we substitute $w=(y, s / \sqrt{|s|})$, using also $n+2+\eta>0$, and we obtain

$$
\begin{aligned}
J_{2} & \leq C(\xi) \rho(x, t)^{\xi} \int_{|w| \leq 3 \rho(x, t)} 2\left(1+|w|^{2}\right)^{\eta / 2}|w| d w \\
& \leq C(\xi) \rho(x, t)^{\xi} \int_{|w| \leq 3 \rho(x, t)}(1+|w|)^{\eta+1} d w \\
& \leq C(\xi) \rho(x, t)^{\xi} C(n, \eta)(1+3 \rho(x, t))^{n+2+\eta} \leq C(n, \xi, \eta) \rho(x, t)^{n+2+\xi+\eta} .
\end{aligned}
$$

Finally, in $R_{3}$ we have

$$
\sigma(x-y, t-s) \leq \sigma(x, t)+\sigma(y, s) \leq \rho(x, t)+\rho(y, s) \leq \frac{1}{2} \sigma(x-y, t-s)+\rho(y, s),
$$

and hence $\sigma(x-y, t-s) \leq 2 \rho(y, s)$. We replace $\rho(y, s)$ with $\sigma(x-y, t-s)$ in (8.8), substitute $z=y-x, r=s-t$, and find that

$$
J_{3} \leq C(\eta) \int_{\sigma(z, r) \geq 2 \rho(x, t)} \sigma(z, r)^{\xi+\eta} d z d r .
$$

Then, as in $J_{1}$ we set $w=(z, r / \sqrt{|r|})$, recalling that $n+2+\xi+\eta<0$, and compute

$$
J_{3} \leq C(\eta) \int_{|w| \geq \rho(x, t)}|w|^{\xi+\eta+1} d w=C(n, \xi, \eta) \rho(x, t)^{n+2+\xi+\eta} .
$$

Proof of Lemma 2.2. First we prove inequality (2.9) for $\mathcal{S} f$. We may assume $f \geq 0$, since $|\mathcal{S} f| \leq \mathcal{S}(|f|)$; then $\mathcal{S} f \geq 0$.

First consider the case $1<p \leq \infty$. Define $q$ as usual by $1 / p+1 / q=1$, and let $\tau$ be a real number to be specified later. Define functions $Y(y)=y, S(s)=s$. By Hölder's inequality,

$$
\begin{aligned}
\mathcal{S} f(x, t) & =\int_{\mathbb{R}^{n+1}} \sigma(x-y, t-s)^{-n / p} f(y, s) \rho(y, s)^{-\tau} \sigma(x-y, t-s)^{-n / q} \rho(y, s)^{\tau} d y d s \\
& \leq\left\|\sigma(x-Y, t-S)^{-n / p} f \rho^{-\tau}\right\|_{p}\left\|\sigma(x-Y, t-S)^{-n / q} \rho^{\tau}\right\|_{q} .
\end{aligned}
$$

We choose $\tau$ so that

$$
-n-2<\tau q<-2
$$

then by Lemma 8.1, noting that

$$
\left\|\sigma(x-Y, t-S)^{-n / q} \rho^{\tau}\right\|_{q}=J(x, t, n,-n, \tau q)^{1 / q},
$$

we obtain

$$
\mathcal{S} f(x, t) \leq C(n, p, \tau)\left\|\sigma(x-Y, t-S)^{-n / p} f \rho^{-\tau}\right\|_{p} \rho(x, t)^{\tau+2 / q} .
$$

If $p=\infty, q=1$, then (2.8) gives $0<\delta<n$, and we may choose $\tau=-\delta-2$ in (8.9); then (8.10) yields

$$
\rho(x, t)^{\delta} \mathcal{S} f(x, t) \leq C(n, p, \delta)\left\|\rho^{\delta+2} f\right\|_{\infty},
$$


and thereby (2.9) with $p=\infty$. When $1<p<\infty$, we obtain instead that

$$
\begin{aligned}
& \left(\left\|\rho^{\delta} \mathcal{S} f\right\|_{p}\right)^{p}=\int_{\mathbb{R}^{n+1}} \rho(x, t)^{\delta p} \mathcal{S} f(x, t)^{p} d x d t \\
& \leq C(n, p, \tau) \int_{\mathbb{R}^{n+1}} \rho(x, t)^{\delta p}\left(\left\|\sigma(x-Y, t-S)^{-n / p} f \rho^{-\tau}\right\|_{p}\right)^{p} \rho(x, t)^{\tau p+2 p / q} d x d t \\
& =C(n, p, \tau) \int_{\mathbb{R}^{n+1}} f(y, s)^{p} \rho(y, s)^{-\tau p} J(y, s, n,-n, \delta p+\tau p+2 p / q) d y d s .
\end{aligned}
$$

We assume further that

$$
-n-\delta p-2 p<\tau p<-\delta p-2 p
$$

then Lemma 8.1 yields

$$
J(y, s, n,-n, \delta p+\tau p+2 p / q) \leq C(n, p, \tau, \delta) \rho(y, s)^{\tau p+\delta p+2 p},
$$

and we obtain

$$
\left(\left\|\rho^{\delta} \mathcal{S} f\right\|_{p}\right)^{p} \leq C(n, p, \tau, \delta) \int_{\mathbb{R}^{n+1}} f(y, s)^{p} \rho(y, s)^{(\delta+2) p} d y d s
$$

This inequality gives (2.9), provided we can confirm that $\tau=\tau(n, p, \delta)$ can be chosen so that (8.9) and (8.11) hold. But these inequalities reduce to the pair

$$
-\frac{n+2}{q}<\tau<-\frac{2}{q}, \quad-\delta-2-\frac{n}{p}<\tau<-\delta-2,
$$

which have a common solution $\tau$ if and only if

$$
-\frac{n+2}{q}<-\delta-2 \text { and }-\delta-2-\frac{n}{p}<-\frac{2}{q}
$$

These last inequalities simplify to our assumption on $\delta$ in (2.8).

Finally, in the case $p=1$ we have

$$
\left\|\rho^{\delta} \mathcal{S} f\right\|_{1}=\int_{\mathbb{R}^{n+1}} \rho(x, t)^{\delta} \mathcal{S} f(x, t) d x d t=\int_{\mathbb{R}^{n+1}} f(y, s) J(y, s, n,-n, \delta) d y d s .
$$

Now (2.8) becomes $-n-2<\delta<-2$, and Lemma 8.1 yields

$$
J(y, s, n,-n, \delta) \leq C(n, \delta) \rho(y, s)^{\delta+2} ;
$$

thus we obtain the required inequality

$$
\left\|\rho^{\delta} \mathcal{S} f\right\|_{1} \leq C(n, \delta) \int_{\mathbb{R}^{n+1}} f(y, s) \rho(y, s)^{\delta+2} d y d s=C(n, \delta)\left\|\rho^{\delta+2} f\right\|_{1},
$$

completing the proof of (2.9) for $\mathcal{S} f$.

In order to verify $(2.9)$ for $\mathcal{K} f$, we use (5.4) to observe that

$$
|\mathcal{K} f| \leq \mathcal{K}(|f|) \leq C(n) \mathcal{S}(|f|) ;
$$

thus the result for $\mathcal{K}$ follows from that for $\mathcal{S}$. 


\section{REFERENCES}

1. H. Begehr and G. N. Hile, Schauder estimates and existence theory for entire solutions of linear elliptic equations, Proc. of Royal Soc. Edinburgh 110A (1988), 101-123. MR 90a:35004

2. M. Cantor, Spaces of functions with asymptotic conditions on $\mathbb{R}^{n}$, Indiana Univ. Math. J. 24 (1975), 897-902. MR 51:1873

3. A. Friedman, Partial Differential Equations of Parabolic Type, Prentice-Hall, Englewood Cliffs, N.J., 1964. MR 31:6062

4. D. Gilbarg and N. S. Trudinger, Elliptic Partial Differential Equations of Second Order, 2nd ed., Springer-Verlag, Berlin, 1983. MR 86c:35035

5. G. N. Hile and C. P. Mawata, Liouville theorems for nonlinear elliptic equations of second order, Partial Differential Equations with Real Analysis, Pitman Research Notes in Math. (H. Begehr and A Jeffrey, eds.) 263 (1992), 57-92. MR 93m:35016

6. G. N. Hile and C. P. Mawata, Liouville theorems for nonlinear parabolic equations of second order, Differential and Integral Equations 9 (1) (1996), 149-172. MR 96k:35085

7. T. Kato, Perturbation Theory for Linear Operators, 2nd ed., Springer-Verlag, Berlin, 1976. MR 53:11389

8. R. B. Lockhart, Fredholm properties of a class of elliptic operators on non-compact manifolds, Duke Math. J. 48 (1981), 289-312. MR 82j:35050

9. R. B. Lockhart and R. C. McOwen, On elliptic systems in $\mathbb{R}^{n}$, Acta Math. 150 (1983), 125-135; 153 (1984), 303-304. MR 84d: 35048; MR 86a:35049

10. C. P. Mawata, Schauder estimates and existence theory for entire solutions of linear parabolic equations, Differential and Integral Equations 2 (3) (1989), 251-274. MR 90i:35024

11. R. C. McOwen, Behavior of the Laplacian on weighted Sobolev spaces, Comm. Pure Appl. Math. 32 (1979), 783-795. MR 81m:47069

12. R. C. McOwen, On elliptic operators in $\mathbb{R}^{n}$, Comm. Partial Differential Equations 5 (1980), 913-933. MR 84k:35058

13. L. Nirenberg and H. F. Walker, The null spaces of elliptic partial differential operators in $\mathbb{R}^{n}$, J. Math. Anal. Appl. 42 (1973), 271-301. MR 47:9354

14. H. F. Walker, On the null-spaces of first order elliptic partial differential operators in $\mathbb{R}^{n}$, Proc. Amer. Math. Soc. 30 (1971), 278-286. MR 43:6583

15. H. F. Walker, On the null-spaces of elliptic partial differential operators in $\mathbb{R}^{n}$, Trans. Amer. Math. Soc. 173 (1972), 263-275. MR 46:7972

16. D. V. Widder, The Heat Equation, Academic Press, New York, San Francisco, London, 1975. MR 57:6840

17. D. V. Widder, Series expansions of solutions of the heat equation in $n$ dimensions, Ann. Mat. Pura Appl. 55 (1961), 389-409. MR 25:331

University of Hawait, Manoa, 2565 The Mall, Honolulu, Hawail 96822

E-mail address: hile@math.hawaii.edu

University of Tennessee, Chattanooga, 615 McCallie Avenue, Chattanooga, TenNESSEE 37403

E-mail address: cmawata@cecasun.utc.edu 